\title{
Central Hepatectomy in a 6-Month-Old Child with Hepatoblastoma following Chemotherapy
}

\author{
Huu Thien $\mathrm{Ho}^{\mathrm{a}}$ Trung Hieu Mai ${ }^{\mathrm{a}}$ Thanh Xuan Nguyen ${ }^{\mathrm{a}}$ \\ Kim Hoa thi Nguyen ${ }^{b}$ Nhu Hien Pham ${ }^{a}$ Huu Son Nguyen ${ }^{b}$ \\ aDepartment of Abdominal Emergency and Pediatric Surgery, Hue Central Hospital,

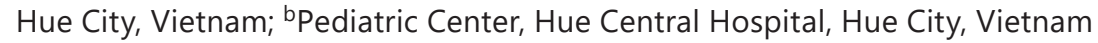

\section{Keywords}

Hepatectomy $\cdot$ Hepatoblastoma $\cdot$ Small child

\begin{abstract}
A hepatoblastoma in a 6-month-old child was initially considered unresectable because of diffuse liver involvement. The patient received 4 courses of cisplatin with an interval time of 2 weeks. A computed tomography scan after 4 courses of chemotherapy showed shrinking of the tumor, which made it resectable, and the tumor was removed by central hepatectomy. The patient was able to eat a regular diet on the fourth day and was sent home on the seventh day, after the operation. The pediatric oncologist followed the patient with liver ultrasonography and alpha-fetoprotein and administered 2 more cycles of cisplatin.
\end{abstract}

\section{(c) 2021 The Author(s).}

Published by S. Karger AG, Basel

\section{Introduction}

Hepatoblastoma is the most common malignancy of the liver in children. The incidence rate of hepatoblastoma is about 1.2/1,000,000, and about 100 new cases are diagnosed every year in the USA [1]. The incidence of hepatoblastoma has increased over the past 2 decades, partly due to increased survival rates of premature and low-birth-weight infants [2-5]. Hepatoblastoma often presents with a large abdominal mass and elevated $\alpha$-fetoprotein (AFP) values and mainly affects children under 3 years of age. Surgery still plays an important role in controlling hepatoblastoma, and complete removal is the only way to cure the malignancy [6]. In addition, the introduction of platinum-based chemotherapy regimens has significantly improved the outcome of hepatoblastoma [7].

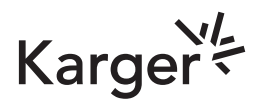



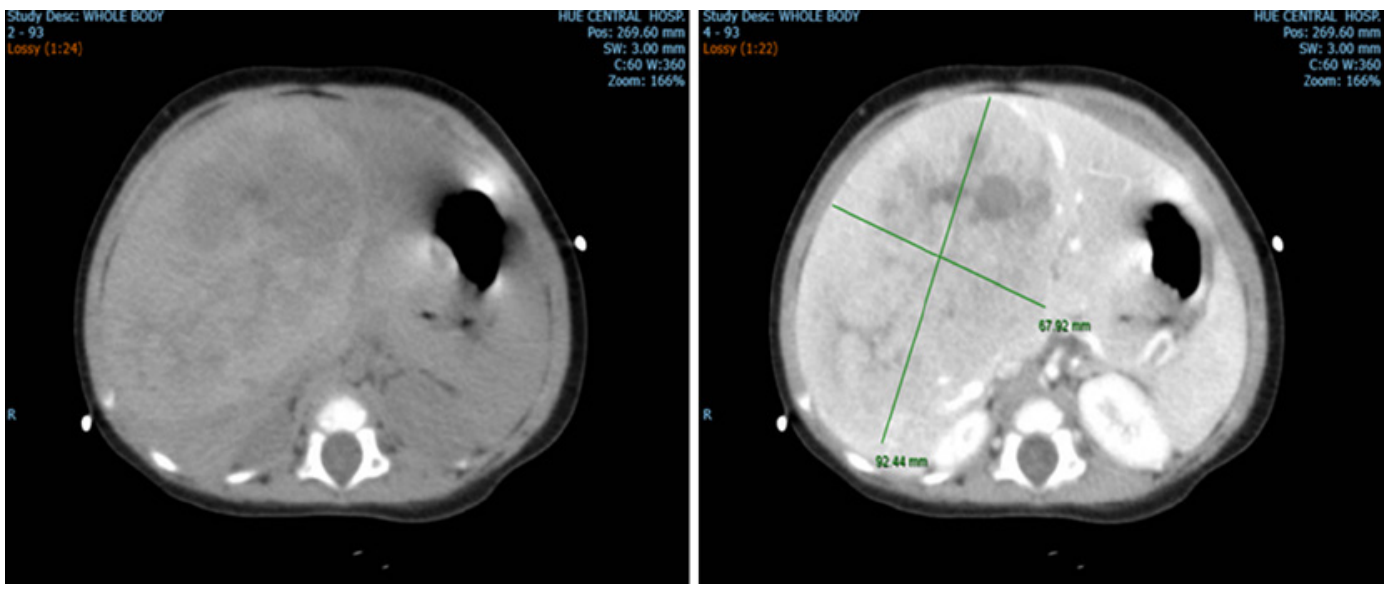

Fig. 1. CT scan at admission showing a heterogeneous mass measuring $9 \times 26 \times 88 \mathrm{~cm}$ at segment IV-V-VIII without metastasis. CT, computed tomography.

Fig. 2. Needle biopsy 1 week after admission showing liver cells resembling fetal hepatocytes with round nuclei.

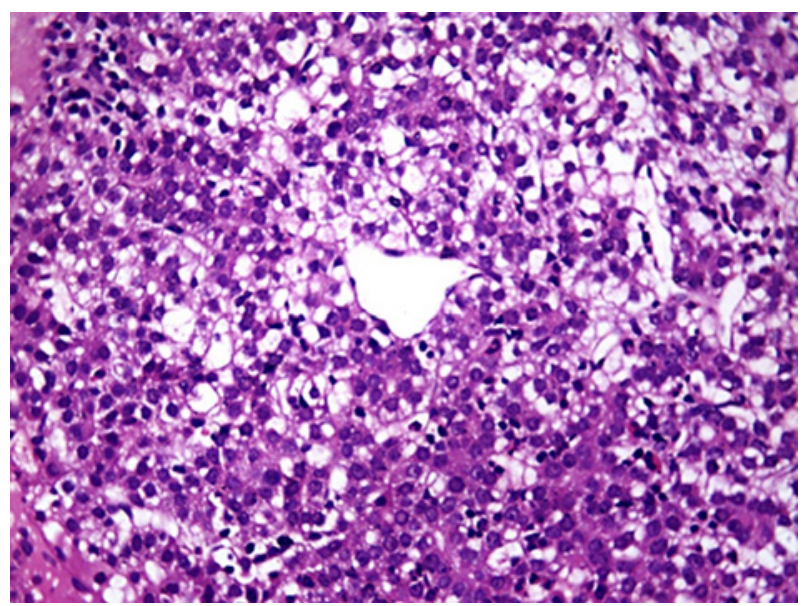

Central hepatoblastomas (CHBLs) involving liver segments (IV + V) or (IV + V + VIII) are in contact with the portal bifurcation. The resection of CHBL can be achieved by central hepatectomy $(\mathrm{CH})$ with thin resection margins on both sides of the liver pedicle or by extended right or left hepatectomy with thin resection margins on one side [8]. Surgical techniques and surgical tools have advanced in the past decades, making precise liver cutting much more favorable and allowing surgeons to perform complex liver resections. In this paper, we report on a 6-month-old girl with CHBL who was successfully treated by $\mathrm{CH}$ following neochemotherapy.

\section{Case Report}

A 2-month-old girl, $4.7 \mathrm{~kg}$ in weight, was hospitalized due to vomiting and blood in her stool. A physical examination found hepatomegaly. At admission, an ultrasound and computed tomography (CT) scan discovered 1 heterogeneous mass measuring $9.2 \times 6.8 \times 8 \mathrm{~cm}$ at the segment IV- V- VIII without metastasis (Fig. 1). The AFP level was $>1,000 \mathrm{IU} / \mathrm{mL}$. Biopsy showed liver cells resembling fetal hepatocytes with round nuclei (Fig. 2). Immunohistochemistry showed the following: CK AE1/3: positive; AFP, Hep-Par 1: positive; CK7, TTF1, 
Fig. 3. CT scan after 4 courses of neochemotherapy showing the shrinking of the tumor, which made it resectable. CT, computed tomography.

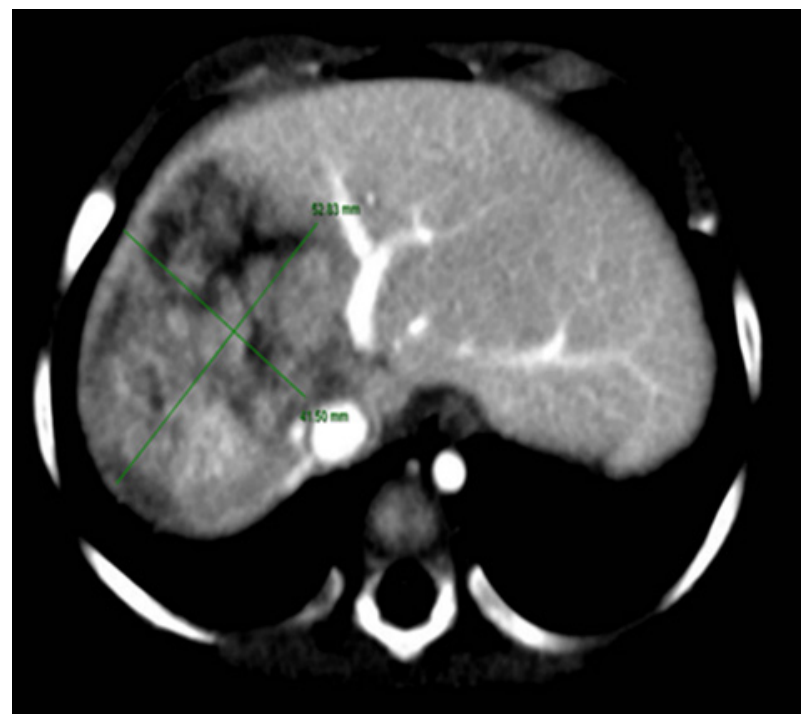

Fig. 4. The IV, V, and VIII segments of the specimen.

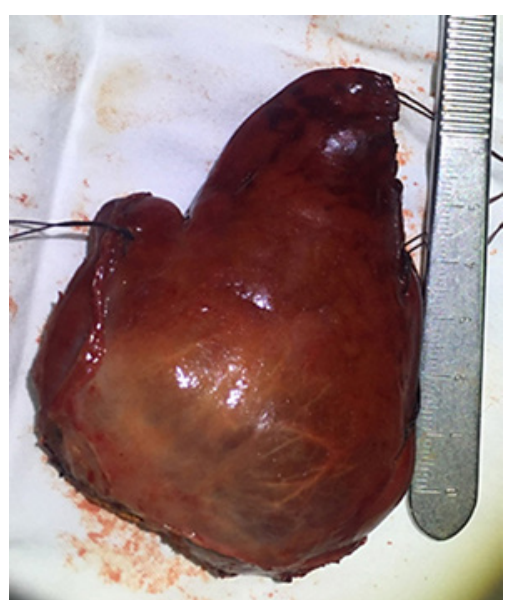

chromogranin: negative; Ki67: positive. The diagnosis was hepatoblastoma of the embryonal type, pretreatment extent of tumor (PRETEXT) III, with standard risk.

The patient received 4 courses of cisplatin with an interval time of 2 weeks. CT scan after 4 courses of chemotherapy showed shrinking of the tumor, which made the tumor resectable (Fig. 3). CH was planned and performed using a midline incision, and the liver was mobilized. The biliary vesicle was dissected and used as traction for hepatic duct dissection. The hepatoduodenal ligament was surrounded by vascular tape in preparation for a Pringle's maneuver. The right and left hepatic ducts, arteries, and portal veins were isolated. The inflow pedicles to segment 4 were isolated, followed by the right anterior sectoral pedicle. The parenchymal transection using Sonastar (Pharmed UK) proceeded from the right side to the left side until the 2 planes met just anterior to the inferior vena cava. The control of the arterial and portal inflow to segments IV and the right anterior sector were accomplished via ligation when the sector was clearly defined. The middle hepatic vein was ligated as the dissection approached the vena cava, and the right hepatic vein was preserved. Segments 4, 5, and 8 were removed en masse (Fig. 4, 5). The resection faces were covered by bioglue.

The operating time was $5 \mathrm{~h} 30$ min (pedicle dissection: $2 \mathrm{~h}$; parenchymal dissection: $2 \mathrm{~h}$ and $30 \mathrm{~min}$ ). The patient was operated on under vascular exclusion of the pedicles. 


\section{Case Reports in Oncology}

\begin{tabular}{l|l}
\hline Case Rep Oncol 2021;14:874-880 \\
\hline DOI: 10.1159/000516800 & $\begin{array}{l}\text { ○ 2021 The Author(s). Published by S. Karger AG, Basel } \\
\text { www.karger.com/cro }\end{array}$ \\
\hline
\end{tabular}

Ho et al.: Central Hepatectomy in Hepatoblastoma

Fig. 5. The IV, V, and VIII segments of the specimen (split).

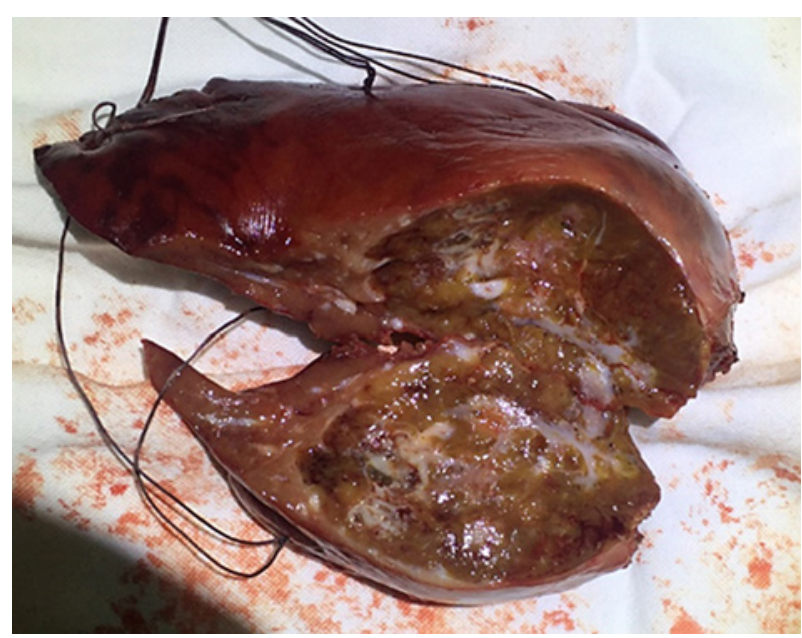

Fig. 6. Color change after RAP and IV segment clamps.

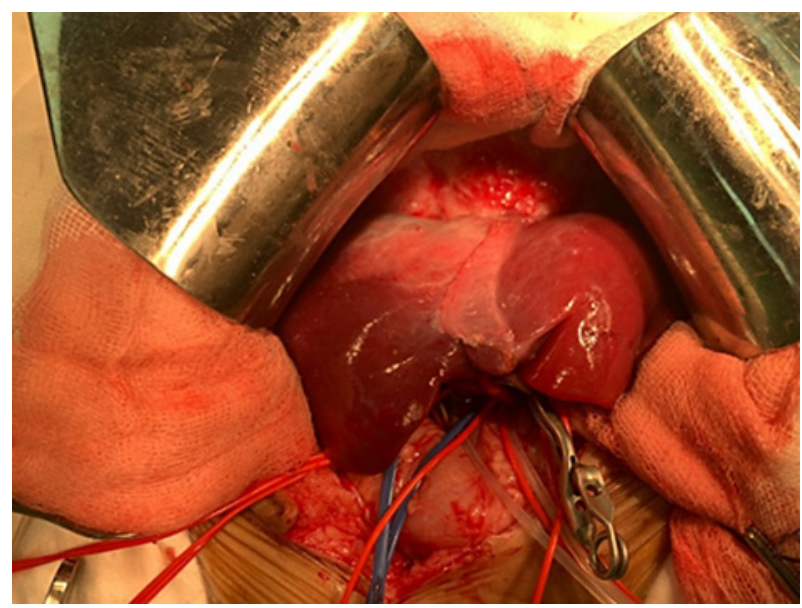

Fig. 7. The remaining right posterior and left lateral segments.

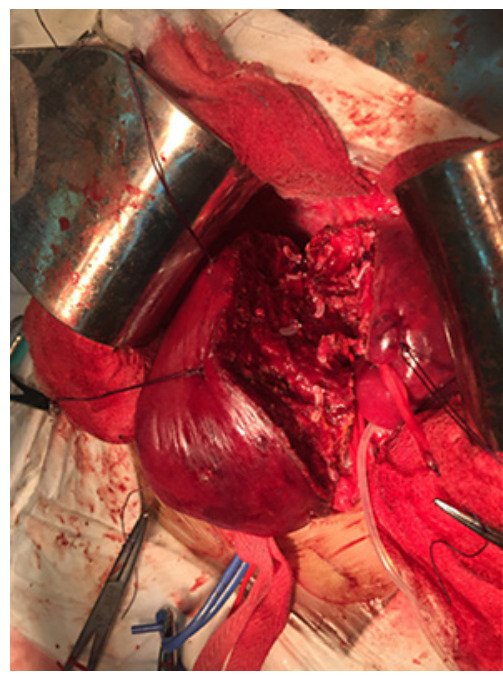

Our patient did not require intraoperative ultrasonography. The amount of blood transfused during surgery was $250 \mathrm{~mL}$. The intraoperative aspects of surgery are shown in Figures 6 and 7. 
The patient was observed in the recovery room for respiration, hemorrhagic syndrome, biliary leakage, or liver failure. Abdominal drainage was left in place to assess postoperative hemorrhaging. The mechanical ventilation was removed the day after surgery. Postoperative recovery was excellent. The aspartate transaminase and alanine aminotransferase levels reduced quickly from 574 and $294 \mathrm{U} / \mathrm{L}$ on the first day to 82 and $95 \mathrm{U} / \mathrm{L}$ on the third day, respectively. The blood count showed a slight increase in white blood cell count $(11.4 \mathrm{k} / \mathrm{mcL})$. The prothrombin time ratio remained low at $44 \%$ from the first day to the third day and increased steadily through the fourth and fifth days (59 and $65.7 \%$, respectively), finally reaching $85 \%$ on the discharge day. A light pink fluid flowed through the drain at about 30 $\mathrm{mL}$ per day, and the drain was removed on the fourth day. The patient was able to eat a regular diet on the fourth day after the operation and was sent home on the seventh day. The pediatric oncologist followed the patient with liver ultrasonography and AFP and administered 2 more cycles of cisplatin.

\section{Discussion}

Treatment abandonment in children with cancer is not unusual in developing countries, especially in children with end-stage cancer [9]. To improve the management and outcomes of hepatoblastoma in developing countries, such experiences are worth reporting.

The most useful diagnostic modality is multiphase CT or magnetic resonance imaging. The helical CT findings of hypervascular lesions in the liver with delayed contrast excretion are highly suggestive of a malignant liver tumor. Histological diagnosis of a tumor specimen is essential, although some investigators believe that biopsy may not be necessary for young children (6 months-3 years) with very high AFP levels [10]. Moreover, a diagnostic surgical biopsy is strongly recommended for all patients and is mandatory for children under 6 months of age due to the wide range of possible tumors presenting at this age, as well as the possible confounding effects of an elevated AFP level due to the age of the child. In addition, at this young age, we need to differentiate hepatoblastomas from rhabdoid tumors [11]. Our patient was admitted at 2 months old, which is why we performed a needle biopsy on her.

In recent years, better diagnostic methods have made possible the earlier diagnosis of hepatic neoplasms and improved techniques for hepatic resection have increased the number of resectable tumors. However, even today, only about one-half of hepatoblastoma cases are surgically resectable [12]. For this reason, chemotherapy plays a very important role in reducing the size of the tumor to the point of resectability. The SIOPEL 3 protocol is used for patients with standard risk. This protocol has 2 regimens: one regimen uses only cisplatin, while the other regimen uses cisplatin and doxorubicin. Compared with cisplatin and doxorubicin, cisplatin monotherapy achieved similar complete ablation and survival in children with standard-risk hepatoblastoma [13]. To avoid side effects due to chemotherapy, we selected monotherapy with cisplatin for our patient in the present study and adjust the dose of cisplatin for infants with body weights $<5 \mathrm{~kg}$. During chemotherapy, the patient did not experience any special side effects, only mild febrile neutropenia. The prognosis is excellent for children with standard risk who have a good response to chemotherapy and good resection. The 3-years event-free survival and overall survival were observed to be $83-85 \%$ and $93-95 \%$ [13].

Regarding surgery, the number of children treated with $\mathrm{CH}$ is still limited in the literature. The study of Florent Guérin examined the largest series of CHs for HBL [14, 15], with 9 CHs for CHBL, while the study of Li et al. [16] reported only 2/93 (2.2\%) CHs. Primary hepatectomy is recommended for patients with PRETEXT stages I and II without additional annotated risk factors. PRETEXT stage III patients should undergo adjuvant chemotherapy with

\section{Karger'}


delayed surgery. As our patient presented PRETEXT III diagnosis on admission, we chose the neoplastic adjuvant chemotherapy strategy and delayed surgery, as recommended by SIOPEL.

The results of this case in a tertiary polyclinic hospital demonstrated the feasibility and safety of the $\mathrm{CH}$ procedure for young children, as long as the surgeons are equipped with modern surgical instruments and trained in liver surgery. The application of parenchymal dissection using Sonastar led to less bleeding and clearly defined the hepatic pedicles.

Our operating time was $5 \mathrm{~h}$ and $30 \mathrm{~min}$, which is slightly longer than the mean operating time reported by Guérin et al. [8] ( $4 \mathrm{~h}$ and $50 \mathrm{~min}$ ). The blood transfusion during surgery was similar between our report and Guérin's study $(250 \mathrm{~mL})$, which is acceptable for first cases. The postoperative course of our case was also similar to that of Guérin's study [8].

\section{Conclusion}

This case demonstrated the feasibility of using $\mathrm{CH}$ as a neoadjuvant therapy for CHBL in a tertiary polyclinic hospital. This treatment avoided the unnecessary sacrifice of functional parenchyma, which could prevent postoperative liver failure.

\section{Statement of Ethics}

Written informed consent was obtained from the patient's parents for the publication of this case report and the accompanying images.

\section{Conflict of Interest Statement}

The authors declare no conflict of interest in association with this article.

\section{Funding Sources}

There were no funding sources.

\section{Author Contributions}

Ho H.T., Mai T.H., and Nguyen T.X. contributed substantially to the conception and design of the case report, as well as to the acquisition, analysis, and interpretation of the patient data. Ho H.T. and Nguyen T.X. drafted the manuscript. Nguyen T.K.H., Pham N.H., and Nguyen H.S. critically reviewed and revised the manuscript for important intellectual content. All the authors gave their final approval.

\section{References}

1 Darbari A, Sabin KM, Shapiro CN, Schwarz KB. Epidemiology of primary hepatic malignancies in U.S. children. Hepatology. 2003 Sep;38(3):560-6.

2 Allan BJ, Parikh PP, Diaz S, Perez EA, Neville HL, Sola JE. Predictors of survival and incidence of hepatoblastoma in the paediatric population. HPB. 2013 Oct;15(10):741-6.

3 Hung GY, Lin LY, Yu TY, Lee CY, Yen HJ, Horng JL. Hepatoblastoma incidence in Taiwan: a population-based study. J Chin Med Assoc. 2018 Jun;81(6):541-7. 
4 Linabery AM, Ross JA. Trends in childhood cancer incidence in the U.S. (1992-2004). Cancer. 2008 Jan 15; 112(2):416-32.

5 Spector LG, Birch J. The epidemiology of hepatoblastoma. Pediatr Blood Cancer. 2012 Nov;59(5):776-9.

6 Malogolowkin MH, Katzenstein HM, Meyers RL, Krailo MD, Rowland JM, Haas J, et al. Complete surgical resection is curative for children with hepatoblastoma with pure fetal histology: a report from the Children's Oncology Group. J Clin Oncol. 2011 Aug 20;29(24):3301-6.

7 Aronson DC, Meyers RL. Malignant tumors of the liver in children. Semin Pediatr Surg. 2016 Oct;25(5):265-75.

8 Guérin F, Gauthier F, Martelli H, Fabre M, Baujard C, Franchi S, et al. Outcome of central hepatectomy for hepatoblastomas. J Pediatr Surg. 2010 Mar;45(3):555-63.

9 Magrath I, Steliarova-Foucher E, Epelman S, Ribeiro RC, Harif M, Li CK, et al. Paediatric cancer in low-income and middle-income countries. Lancet Oncol. 2013 Mar;14(3):e104-16.

10 Czauderna P, Otte JB, Aronson DC, Gauthier F, Mackinlay G, Roebuck D, et al. Guidelines for surgical treatment of hepatoblastoma in the modern era: recommendations from the childhood liver tumour strategy group of the international society of paediatric oncology (SIOPEL). Eur J Cancer. 2005 May;41(7):1031-6.

11 Dall'Igna P, Brugieres L, Christin AS, Maibach R, Casanova M, Alaggio R, et al. Hepatoblastoma in children aged less than six months at diagnosis: a report from the SIOPEL group. Pediatr Blood Cancer. 2018 Jan;65(1).

12 Fegiz G, Rosati D, Tonelli F, Donfrancesco A. A case report of hepatoblastoma treated by chemotherapy and hepatic lobectomy. World J Surg. 1977 May;1(3):407-14.

13 Perilongo G, Maibach R, Shafford E, Brugieres L, Brock P, Morland B, et al. Cisplatin versus cisplatin plus doxorubicin for standard-risk hepatoblastoma. N Engl J Med. 2009 Oct 22;361(17):1662-70.

14 La Quaglia MP, Shorter NA, Blumgart LH. Central hepatic resection for pediatric tumors. J Pediatr Surg. 2002 Jul;37(7):986-9.

15 Ohno Y, Furui J, Kanematsu T. Is a modified central bisegmentectomy a volume-saving operation for pediatric hepatoblastoma? J Pediatr Surg. 2004 Jan;39(1):E13-6.

16 Li J, Li H, Wu H, Niu H, Li H, Pan J, et al. Outcomes of children with hepatoblastoma who underwent liver resection at a tertiary hospital in China: a retrospective analysis. BMC Pediatr. 2020 May 9;20(1):200. 